\title{
Short-echo-time magnitude image derived from quantitative susceptibility mapping could resemble neuromelanin-sensitive MRI image in substantia nigra
}

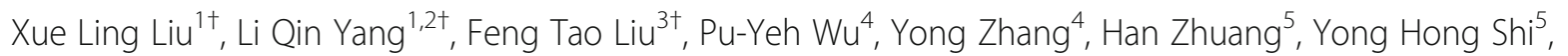
Jian Wang ${ }^{3}$, Dao Ying Geng ${ }^{1 *}$ and Yu Xin Li ${ }^{1 *}$ (D)

\begin{abstract}
Background: In this study, we explored whether the proposed short-echo-time magnitude (setMag) image derived from quantitative susceptibility mapping (QSM) could resemble NM-MRI image in substantia nigra (SN), by quantitatively comparing the spatial similarity and diagnosis performances for Parkinson's disease (PD).

Methods: QSM and NM-MRI were performed in 18 PD patients and 15 healthy controls (HCs). The setMag images were calculated using the short-echo-time magnitude images. Bilateral hyperintensity areas of SN ( $\mathrm{SN}_{\text {hyper }}$ ) were manually segmented on setMag and NM-MRI images by two raters in a blinded manner. The inter-rater reliability was evaluated by the intraclass correlation coefficients (ICC) and the Dice similarity coefficient (DSC). Then the intermodality (i.e. setMag and NM-MRI) spatial similarity was quantitatively assessed using DSC and volume of the consensual voxels identified by both of two raters. The performances of mean $S N_{\text {hyper }}$ volume for PD diagnosis on setMag and NM-MRI images were evaluated using receiver operating characteristic (ROC) analysis.

Results: The $\mathrm{SN}_{\text {hyper }}$ segmented by two raters showed substantial to excellent inter-rater reliability for both setMag and NM-MRI images. The DSCs of SN $\mathrm{N}_{\text {hyper }}$ between setMag and NM-MRI images showed substantial to excellent voxel-wise overlap in HCs $(0.80 \sim 0.83)$ and PD $(0.73 \sim 0.76)$, and no significant difference was found between the $S N_{\text {hyper }}$ volumes of setMag and NM-MRI images in either HCs or PD ( $\left.p>0.05\right)$. The mean $S N_{\text {hyper }}$ volume was significantly decreased in PD patients in comparison with HCs on both setMag images $\left(77.61 \mathrm{~mm}^{3} \mathrm{vs} 95.99 \mathrm{~mm}^{3}\right.$, $p<0.0001)$ and NM-MRI images $\left(79.06 \mathrm{~mm}^{3}\right.$ vs $\left.96.00 \mathrm{~mm}^{3}, p<0.0001\right)$. Areas under the curve (AUCs) of mean $\mathrm{SN}_{\text {hyper }}$ volume for PD diagnosis were 0.904 on setMag and 0.906 on NM-MRI images. No significant difference was found between the two curves $(p=0.96)$.

Conclusions: $\mathrm{SN}_{\text {hyper }}$ on setMag derived from QSM demonstrated substantial spatial overlap with that on NM-MRI and provided comparable PD diagnostic performance, providing a new QSM-based multi-contrast imaging strategy for future PD studies.
\end{abstract}

Keywords: Parkinson disease, Substantia nigra, Pars compacta, Quantitative susceptibility mapping, Neuroimaging

*Correspondence: gengdy@163.com; liyuxin@fudan.edu.cn

†Xue Ling Liu, Li Qin Yang and Feng Tao Liu are contributed equally to this work.

'Department of Radiology, Huashan Hospital, Fudan University, Shanghai 200040, China

Full list of author information is available at the end of the article

(c) The Author(s). 2020 Open Access This article is licensed under a Creative Commons Attribution 4.0 International License, which permits use, sharing, adaptation, distribution and reproduction in any medium or format, as long as you give appropriate credit to the original author(s) and the source, provide a link to the Creative Commons licence, and indicate if changes were made. The images or other third party material in this article are included in the article's Creative Commons licence, unless indicated otherwise in a credit line to the material. If material is not included in the article's Creative Commons licence and your intended use is not permitted by statutory regulation or exceeds the permitted use, you will need to obtain permission directly from the copyright holder. To view a copy of this licence, visit http://creativecommons.org/licenses/by/4.0/. The Creative Commons Public Domain Dedication waiver (http://creativecommons.org/publicdomain/zero/1.0/) applies to the data made available in this article, unless otherwise stated in a credit line to the data. 


\section{Background}

Parkinson's disease (PD) is characterized by the progressive loss of neuromelanin-containing dopaminergic neurons [1] in the substantia nigra pars compacta (SNc). Neuromelanin-sensitive MRI (NM-MRI) technique $[1,2]$ is currently used for imaging SNc with hyperintensity, which has been shown positively related to the quantity of neuromelanin-containing neurons by postmortem study [3]. Another pathological hallmark in PD is iron deposition throughout SN [4]. T2*-weighted imaging based on the paramagnetic magnetic susceptibility of iron, such as susceptibility-weighted imaging (SWI) and quantitative susceptibility mapping (QSM), are commonly used to reveal the iron deposition in $\operatorname{PD}[5,6]$.

Previous evidence suggests the roles of neuromelanin and iron are intricate and it is well-recognized that iron deposition is related to the reduction of neuromelanin in SNc in PD [4]. Several studies [7, 8] in PD have conducted NM-MRI and $\mathrm{T} 2{ }^{*}$-weighted imaging, individually, to assess the neurodegenerative changes in SNc. However, the two-sequence approaches are time consuming and inter-modality registration is needed. If there is a neuroimaging technique with multi-contrast both sensitive to neuromelanin and iron, it could be valuable for the study of pathogenesis associated with PD.

In 2015, Langley et al. [9] proposed a technique with two echoes using GRE sequence to generate two contrasts, the first echo taken as the images sensitive to neuromelanin in SNc and the second echo used to generate the susceptibility images sensitive to iron. As we known, QSM is a reliable quantitative technique for magnetic susceptibility assessment [10]. Based on the magnitude and phase images generated from GRE complex data, susceptibility images could be calculated and used for quantitatively assessment of the iron deposition in $\mathrm{PD}[5,6]$. However, up to now, no study about neuromelanin-sensitive contrast in SNc using QSM has been reported. Empirically, we noticed that there was a pair of hyperintensity crescent-shaped structures in the midbrain on the first several short-echo-time magnitude images from QSM, which resemble the previously reported appearance of SNc on NM-MRI images.

In this study, we proposed a new parameter image based on the short-echo-time magnitude images from QSM, denoted as setMag. We hypothesized that the midbrain hyperintensity areas on setMag and $\mathrm{SNc}$ on NM-MRI images are spatially congruent, and that the morphology changes on setMag images could have comparable diagnostic performance as NM-MRI images in PD. Therefore, we quantitatively evaluated the spatial overlap of hyperintensity area in the midbrain on setMag images and SNc on NM-MRI images, and compared their diagnostic performances in $\mathrm{PD}$, aiming to demonstrate the values of setMag for providing neuromelanin- sensitive contrast and serving as a candidate imaging biomarker in future PD studies.

\section{Methods \\ Subjects}

Eighteen PD patients and age-matched 15 healthy controls (HCs) were recruited in this study. Patients with PD were diagnosed by two experts of extrapyramidal motor disease in the neurology department (Feng Tao Liu and Jian Wang), according to the movement disorder society (MDS) clinical diagnostic criteria for PD [11]. All healthy controls were recruited from the community with no history of neuropsychiatric or neurological diseases. Exclusion criteria were as follows: no history of other neurological/psychiatric disorders including Parkinson-plus syndrome, substance abuse, severe infection, and tremor-related dysmetabolism including thyroid dysfunction and drug toxicity.

The motor dysfunction of PD subjects was further evaluated using the Movement Disorder Society Unified Parkinson's Disease Rating Scale Part III [12] (MDSUPDRS Part III) and Hoehn and Yahr scale [13] in the OFF medication state. Disease duration was calculated as the time between the onset of motor symptoms as reported by subjects and the time for MRI scan. This study was approved by the Ethics Committee of Huashan Hospital, Fudan University (approval No. KY 2016-214). Written consent was obtained from each participant.

\section{Image acquisition}

All MR examinations were performed on a 3.0 T MR750 scanner (GE Healthcare, Milwaukee, WI) equipped with an eight-channel head matrix coil at the Department of Radiology of Huashan Hospital of Fudan University, China. Foam padding was applied to prevent head movement for each participant, and earplugs were provided to reduce scanner noise.

The T1-weighted FSE NM-MRI sequence was as per Sasaki et al. [1], and parameters were as follows: repetition time/echo time $(\mathrm{TR} / \mathrm{TE})=600 / 13 \mathrm{~ms}$, bandwidth $=$ $31.25 \mathrm{kHz}$, flip angle $=145^{\circ}$, field of view $($ FOV $)=240 \times$ $240 \mathrm{~mm}$, matrix size $=512 \times 320$, slice thickness $=1.5$ $\mathrm{mm}$, voxel size $=0.47 \times 0.75 \times 1.5 \mathrm{~mm}^{3}$, number of slices $=16, \mathrm{NEX}=5$, acquisition time $=8: 03 \mathrm{~min}$. The $3 \mathrm{D}$ multi-gradient-echo QSM sequence parameters were as follows: $\mathrm{TR}=41.6 \mathrm{~ms}$, number of echoes $=16$, first $\mathrm{TE}=$ $3.2 \mathrm{~ms}$, TE spacing $=2.4 \mathrm{~ms}$, bandwidth $=62.50 \mathrm{kHz}$, flip angle $=12^{\circ}, \quad$ FOV $=256 \times 256 \mathrm{~mm}$, matrix size $=256 \times$ 256 , slice thickness $=1 \mathrm{~mm}$, voxel size $=1 \times 1 \times 1 \mathrm{~mm}^{3}$, number of slices $=140$, acceleration factor $=2$, acquisition time $=9: 00 \mathrm{~min}$. In addition, in order to exclude other pathological diseases in the mesencephalon, conventional MRI scans including T2-weighted fluidattenuated inversion recovery (FLAIR) and diffusion- 
setMag
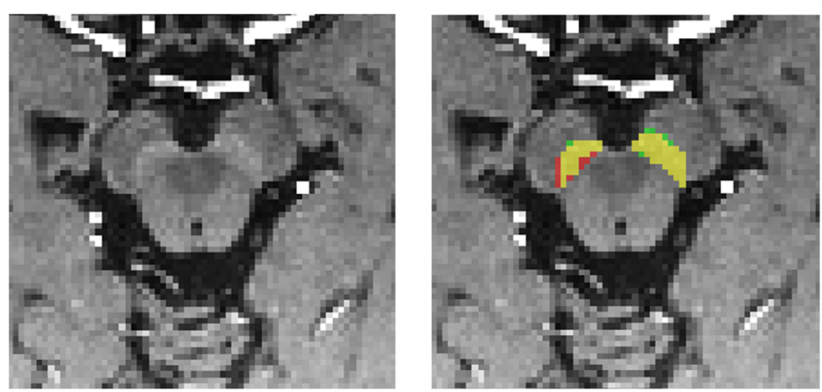

A

$\mathrm{B}$

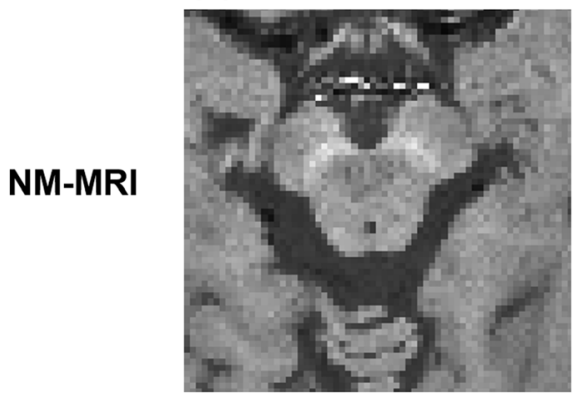

C

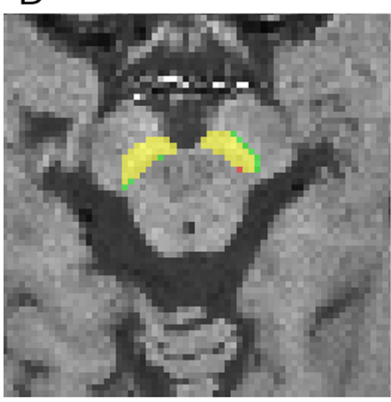

$\mathrm{D}$

Fig. 1 The segmentation of $S N_{\text {hyper }}$ regions of interest (ROI) on setMag and NM-MRI images of a representative subject. A zoomed in view of midbrain area on the setMag and NM-MRI images are shown in $\mathbf{a}$ and $\mathbf{c}$, respectively. The corresponding left and right $\mathrm{SN}_{\text {hyper }}$ regions of interest (ROI) segmented by rater 1 (red), rater 2 (green) and their consensual voxels (yellow) are shown in $\mathbf{b}$ and $\mathbf{d}$

weighted images (DWI) were also acquired prior to the NM-MRI and QSM sequences. All sequences were scanned using axial sections parallel to the anterior commissure-posterior commissure (AC-PC) line, with whole-brain coverage for QSM and the two routine MRI sequences, and with coverage from the upper margin of the mammillary body to the pons for NM-MRI.

\section{Image preprocessing and SN segmentation}

A total of 16 magnitude images (denoted as $\mathrm{Mag}_{\mathrm{i}}$, where i represent number of 1-16) were generated by QSM data for each subject. The first magnitude image, corresponding to the shortest TE, has minimal T2*-weighted contrast and significant T1-weighted contrast especially around $\mathrm{SN}$. As the echo time increases, the contribution of $\mathrm{T}^{*}$-weighted contrast increases gradually [14]. Hypointensity in iron-riched red nucleus (RN), which may represent the emerging of $\mathrm{T} 2 *$ contrast, was shown from $\mathrm{Mag}_{4}$ to $\mathrm{Mag}_{16}$ in our data. Thus, a calculated short-echo-time magnitude image with strengthened T1-weighted contrast, denoted as setMag, was defined using the following formula:

$$
\text { setMag }=\left(\sqrt{\sum\left(M a g_{i}^{2}\right)}\right)^{4}
$$

where $i=1 / 2 / 3$, corresponding to shortest three TEs $(3.2 / 5.6 / 8.0 \mathrm{~ms})$ in this study. The NM-MRI images were co-registered to the setMag images before segmentation. Preprocessing was done using MATLAB (MathWorks, Natick, MA) and Statistical Parametric Mapping (SPM12) (http://www.fil.ion.ucl.ac.uk/spm/).

Hyperintensity areas of substantia nigra $\left(\mathrm{SN}_{\text {hyper }}\right)$ were manually segmented on setMag and NM-MRI images in a blinded manner using ITK-SNAP software [15] by two radiologists with more than five (rater 1, Xue Ling Liu) and 10 (rater 2, Yu Xin Li) years of experience in neuroimaging, respectively. For each subject, left and right $\mathrm{SN}_{\text {hyper }}$ regions of interest (ROIs) were respectively segmented in three consecutive axial slices that internal to cerebral peduncle with best contrast around this region (Fig. 1).

\section{Inter-rater and inter-modality quantitative spatial similarity analysis}

The spatial overlap of each pair of ROIs segmented either by two different raters (i.e. inter-rater) or on two

Table 1 Demographic information and clinical characteristics of healthy controls and PD patients

\begin{tabular}{llll}
\hline Variable & $\mathrm{HCS}(n=15)$ & $\mathrm{PD}(n=18)$ & $p$ \\
\hline Gender (male: female) & $9: 06$ & $7: 11$ & 0.23 \\
Age (median (range), year) & $58(43-66)$ & $61(40-79)$ & 0.09 \\
Disease duration (month) & - & $21.22 \pm 14.60$ & - \\
MDS UPDRS-III score & - & $21.78 \pm 13.96$ & - \\
$\mathrm{H}-\mathrm{Y}$ stage (median (range)) & - & $1(1-3)$ & - \\
\hline
\end{tabular}


Table 2 Inter-rater reliability on setMag and NM-MRI images

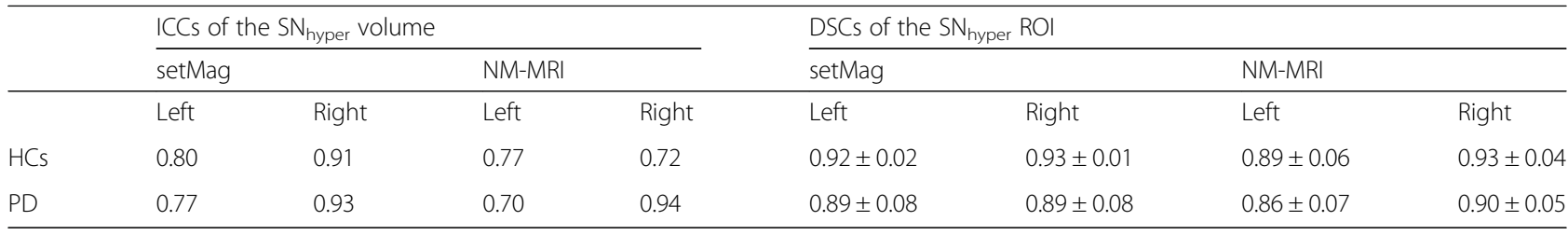

different modalities (setMag and NM-MRI) (i.e. intermodality) was compared using the Dice similarity coefficient (DSC) [16], and was defined as

$$
\mathrm{DSC}=\frac{2 \times V(A \cap B)}{V(A)+V(B)}
$$

$\mathrm{V}$ is the total volume of left or right ROI. Letters A and $\mathrm{B}$ denotes ROIs from different raters or different modalities. The operator $\cap$ represents the intersection area.

\section{Evaluation of diagnostic performance in PD}

The performance of mean volume of left and right $\mathrm{SN}_{\text {hy- }}$ per derived from setMag images in differentiating PD from $\mathrm{HCs}$ were conducted using receiver operating characteristic (ROC) analysis, and was compared with that of NM-MRI images with Medcalc software [17]. The significance level was set at $P<0.05$ (two-tailed).

\section{Statistical analysis}

For comparison of gender between PD and HC groups, chi-square test was used. For age and mean $\mathrm{SN}_{\text {hyper }}$ volume between two groups, data were first tested for normality with the D'Agostino-Pearson omnibus Normality test, then compared using independent samples $t$-tests or Mann-Whitney test. For inter-modality comparison between setMag and NM-MRI images, paired $t$-test or Wilcoxon test were performed. The statistical agreement of $\mathrm{SN}_{\text {hyper }}$ volume between two rates was assessed using the intraclass correlation coefficients (ICC) with a twoway random method, and determined using the following criteria: $(0.8,1]=$ excellent agreement and $(0.6$, 0.8 ] = substantial agreement [18]. The correlations between mean $\mathrm{SN}_{\text {hyper }}$ volume and disease severity (UPDRSIII score and H-Y stage) were used by Spearman correlation analysis. The above statistical analyses were performed using GraphPad Prism Software version 7.0 (GraphPad Prism Software Inc., San Diego, CA).

\section{Results}

Demographic and clinical information

Demographic and clinical information from $\mathrm{HCs}$ and patients with PD are shown in Table 1. There were no significant differences in gender $(p=0.23)$ nor age $(p=$ 0.09 ) between the two groups. Disease duration for PD subjects ranged from 4 to 59 months $(21.22 \pm 14.60$ months), UPDRS-III scores ranged from 4 to 60 $(21.78 \pm 13.96)$ and $\mathrm{H} \& \mathrm{Y}$ score ranged from 1 to 3 (H\&Y 1: $n=10, \mathrm{H} \& \mathrm{Y} 2: n=4$ and H\&Y $3: n=4)$.

\section{Inter-rater reliability on setMag and NM-MRI images}

Table 2 shows the inter-rater reliability of the $\mathrm{SN}_{\text {hyper }}$ segmented by two raters, quantitated by the ICC of the $\mathrm{SN}_{\text {hyper }}$ volume and the DSC of the $\mathrm{SN}_{\text {hyper }} \mathrm{ROIs}$, for setMag or NM-MRI images respectively. All values revealed substantial to excellent inter-rater reliability. Specifically, the ICCs of the $\mathrm{SN}_{\text {hyper }}$ volume were ranged from 0.72 to 0.91 in $\mathrm{HCs}$, and from 0.70 to 0.94 in PD, respectively, and the DSCs of the $\mathrm{SN}_{\text {hyper }} \mathrm{ROI}$ were ranged from 0.89 to 0.93 in $\mathrm{HCs}$, and from 0.86 to 0.90 in PD, respectively.

For following analysis, only the consensual voxels (i.e. the voxels identified by both of two raters) were selected as the left and right $\mathrm{SN}_{\text {hyper }}$ ROIs of setMag and NMMRI images for each subject.

\section{$\mathrm{SN}_{\text {hyper }}$ similarities between setMag and NM-MRI images}

$\mathrm{SN}_{\text {hyper }}$ similarities between setMag and NM-MRI images were estimated through both DSC and volume. As shown in Table 3, the left, right and mean $\mathrm{SN}_{\text {hyper }}$ ROIs between setMag and NM-MRI images have substantial to excellent spatial overlap in either $\mathrm{HC}$ or $\mathrm{PD}$ group, with DSCs of $0.80 \pm 0.05$ and $0.83 \pm 0.04$ for left and right $\mathrm{SN}_{\text {hyper }}$ in $\mathrm{HCs}$, and $0.76 \pm 0.09$ and $0.73 \pm 0.07$ for left and right $\mathrm{SN}_{\text {hyper }}$ in $\mathrm{PD}$, respectively. Besides, there

Table 3 The DSC and volume of consensual voxels identified by both of two raters

\begin{tabular}{|c|c|c|c|c|c|}
\hline Group & & & Left & Right & Mean \\
\hline \multirow[t]{4}{*}{$\mathrm{HCs}$} & DSC & & $0.80 \pm 0.05$ & $0.83 \pm 0.04$ & $0.81 \pm 0.04$ \\
\hline & Volume & setMag & $97.67 \pm 11.34$ & $94.33 \pm 12.61$ & $95.99 \pm 10.60$ \\
\hline & & NM-MRI & $94.53 \pm 11.11$ & $97.47 \pm 9.32$ & $96.00 \pm 8.49$ \\
\hline & & $p$ & 0.45 & 0.31 & 0.99 \\
\hline \multirow[t]{4}{*}{ PD } & DSC & & $0.76 \pm 0.093$ & $0.73 \pm 0.067$ & $0.74 \pm 0.07$ \\
\hline & Volume & setMag & $83.28 \pm 14.54$ & $71.94 \pm 16.73$ & $77.61 \pm 13.06$ \\
\hline & & NM-MRI & $79.78 \pm 13.45$ & $78.33 \pm 15.99$ & $79.06 \pm 11.93$ \\
\hline & & p & 0.36 & 0.053 & 0.62 \\
\hline
\end{tabular}

$\mathrm{p}$ represents the comparison of the volume between $\mathrm{SN}_{\text {hyper }}$ of setMag and NM-MRI images using paired t-test or Wilcoxon test 

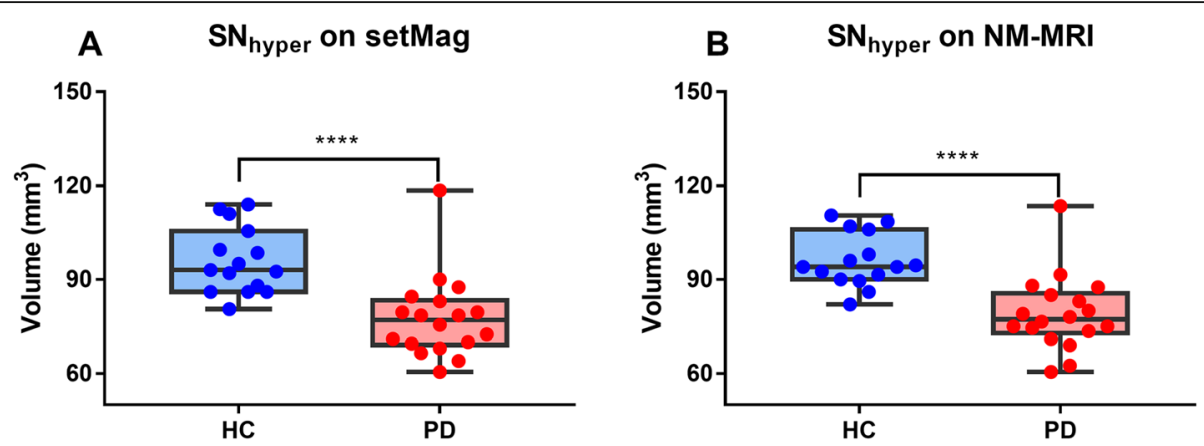

Fig. 2 Comparison of the mean $\mathrm{SN}_{\text {hyper }}$ volume on setMag and NM-MRI images. Comparison of the mean $\mathrm{SN}_{\text {hyper }}$ volume on both setMag (A) and NM-MRI (B) images for HCs and PD patients. The scatter-box diagram denotes the 25th and 75th percentiles with the line denoting the mean value. Significant differences between PD and HCs are represented as: ${ }^{*}{ }^{* *} P<0.0001$

were no significant differences between the mean $\mathrm{SN}_{\mathrm{hy}}$ per volumes of setMag and NM-MRI images in either HCs $(p=0.45$ for left, and $p=0.31$ for right $)$ or PD ( $p=$ 0.36 for left, and $p=0.053$ for right) group.

\section{Diagnostic performance in PD}

The mean $\mathrm{SN}_{\text {hyper }}$ volume was significantly decreased in PD patients compared with HC group on both setMag images $\left(77.61 \pm 13.06 \mathrm{~mm}^{3}\right.$ vs $95.99 \pm 10.60 \mathrm{~mm}^{3}, p<$ $0.0001)$ and NM-MRI images $\left(79.06 \pm 11.93 \mathrm{~mm}^{3}\right.$ vs $96.00 \pm 8.49 \mathrm{~mm}^{3}, p<0.0001$ ) (Fig. 2).

ROC analysis demonstrated that the areas under the curve (AUCs) of mean $\mathrm{SN}_{\text {hyper }}$ volume for discriminating PD from HCs were 0.904 and 0.906 on setMag and NMMRI images, respectively. The sensitivity, specificity and accuracy of the mean $\mathrm{SN}_{\text {hyper }}$ volume on setMag were $83.33,93.33$ and $87.88 \%$ with the optimal cutoff value of $84.50 \mathrm{~mm}^{3}$, and on NM-MRI images were $88.89,86.67$ and $87.88 \%$ with the optimal cutoff value of $88.00 \mathrm{~mm}^{3}$, respectively (Table 4$)$. There was no significant difference $(p=0.96)$ between the two ROC curves for differentiating PD from $\mathrm{HCs}$ (Fig. 3).

\section{Correlation between mean $\mathrm{SN}_{\text {hyper }}$ volume and disease severity}

There was no significant correlation between the mean $\mathrm{SN}_{\text {hyper }}$ volume and UPDRSIII score (setMag: $r=0.33$, $p=0.17$; NM-MRI: $r=-0.07, p=0.78$ ) or H-Y stage (setMag: $r=0.24, p=0.35$; NM-MRI: $r=-0.17, p=0.51$ ).

\section{Discussion}

In this study, we recalculated the setMag images derived from multiple short-echo-time magnitude images of QSM data and found a high spatial similarity (DSC > 0.7 ) between $\mathrm{SN}_{\text {hyper }}$ on setMag and SNc on NM-MRI for both HCs and PD patients, whose volumes could differentiate PD from $\mathrm{HCs}$ without significant intermodality difference. Radiological-histological study has confirmed the co-localization of the hyperintensity area on NM-MRI and neuromelanin-containing dopaminergic neurons in SNc [3]. Our results therefore demonstrated that the proposed setMag image could provide neuromelanin contrast by showing substantially similar location of hyperintensity area with SNc on NM-MRI, and comparable PD diagnostic performance with NMMRI.

The mechanism underlying the contrast of hyperintensity neuromelanin-sensitive area on setMag image may be related with several factors. First, neuromelanin in SNc has specified structural and physical characteristics. Neuromelanin is a macromolecule composed of melanin, proteins, lipids and metal ions [19]. According to in vitro studies in synthetic melanin, the presence of ferric iron in the iron-melanin complex shortened T1 and T2 relaxation times determined by MRI $[20,21]$. Thus, although imaging of macromolecules with short T2 is difficult using standard MR sequences [22], specialized sequences utilizing the short $\mathrm{T} 1$ and other macromolecule-related characteristics could work. Second, accordingly, the scanning parameters of previous NM-MRI techniques $[1,2]$, such as repetition time (TR),

Table 4 Receiver operating characteristic analysis of setMag and NM-MRI for the differentiation of PD patients from healthy controls

\begin{tabular}{llllll}
\hline & AUC & Cut-off value $\left(\mathrm{mm}^{3}\right)$ & Sensitivity (\%) & Specificity (\%) & Accuracy (\%) \\
\hline setMag & 0.904 & $\leq 84.50$ & 83.33 & 93.33 & 87.88 \\
NM-MRI & 0.906 & $\leq 88.00$ & 88.89 & 86.67 & 87.88 \\
\hline
\end{tabular}

$P$ value is the AUC comparison for mean $\mathrm{SN}_{\text {hyper }}$ volume to differentiate $\mathrm{PD}$ from $\mathrm{HCs}$ on setMag and NM-MRI images 


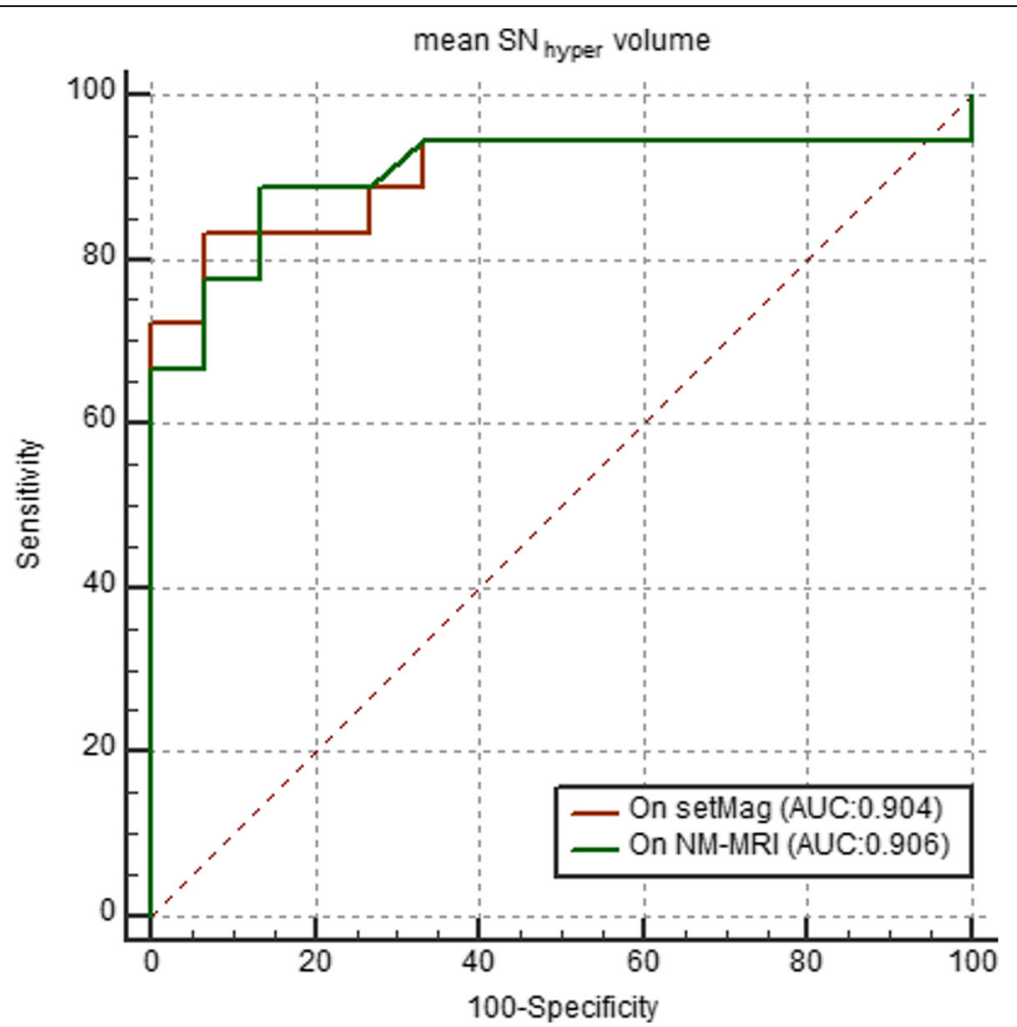

Fig. 3 Receiver operator characteristic analyses of the mean $S N_{\text {hyper }}$ volume for differentiating PD from HCs on setMag and NM-MRI images. There was no significant difference between ROC curves of mean $S N_{\text {hyper }}$ volume $(P=0.96)$ on setMag and NM-MRI images

echo time (TE) and bandwidth, were designed to provide best neuromelanin contrast. The contrasts were mainly based on $\mathrm{T} 1$ effects [20] and magnetization transfer (MT) effects [9]. An in vitro study in synthetic melanins [20] confirmed the role of T1 relaxation time reduction in the mechanism underlying contrast on NM-MRI. The first published NM-MRI study in SNc used a T1weighted fast spin echo sequence (FSE) [1]. As an inherent MT effect was also included in this technique, other studies used explicit MT-MRI sequence and demonstrated the significant contribution of MT effect in neuromelanin-sensitive contrast, in addition to T1effects [9]. In our study, whilst the NM-MRI were conducted using similar parameters as the previous study [1], the setMag images were generated from the short echo time magnitude images of GRE sequence with a short TR, short TE and small flip angle. Such parameters would produce images with minimal $\mathrm{T} 2 *$ contrast and significant $\mathrm{T} 1$ contrast especially between the short $\mathrm{T} 1$ neuromelanin in $\mathrm{SNc}$ and surrounding tissues. Due to the small flip angle, the contribution of implicit MT effect may be neglectable compared with other MT-based NM-MRI [9]. Thus, the neuromelanin-based contrast on our setMag images may mainly attributed to T1 effect. Finally, we performed an exponential transform in calculation of the setMag images, which nonlinearly strengthened the contrast ratio between neuromelanincontaining area and other tissues.

The volume reduction of $\mathrm{SN}_{\text {hyper }}$ in PD patients was mainly related with the decreased neuromelanin during the neurodegenerative process. Neuromelanin could play neuroprotective or neurodegenerative roles in dopaminergic neurons [23]. In normal condition, neuromelanin provide neuronal protect by consuming excess cytosolic dopamine and chelating ferric irons [24]. In cases of iron overload such as PD, excess irons not chelated by neuromelanin could cause cell death by oxidative stress. Afterwards, neuromelanin released by dying neurons is phagocytosed and degraded by microglia, results in a decreased amount. Additionally, irons and other toxins previously accumulated by neuromelanin are released, resulting in a self-propelling mechanism of neuroinflammation and neurodegeneration in PD [23]. The significantly reduced volume of hyperintensity area in $\mathrm{SNc}$ of $\mathrm{PD}$, as revealed by our results of neuromelanin-sensitive setMag and NM-MRI, could be a representation of this cumulative effect of neuromelanin reduction, and used as a convincible biomarker for exhibiting neuromelanin degeneration in PD.

However, no correlation was found between the mean $\mathrm{SN}_{\text {hyper }}$ volume and disease severity (UPDRSIII score or $\mathrm{H}-\mathrm{Y}$ stage) in our results. As previous studies showed 
controversial results, with weak [25] or no correlation [26-28] with UPDRSIII score or H-Y stage, the volume of $\mathrm{SN}_{\text {hyper }}$ as a monitoring tool for PD patients could not be determined and needs more evidence.

There are some limitations in the present study. First, as the spatial resolution of setMag and NM-MRI images was not exactly same, we performed co-registration of NM-MRI to setMag images using SPM12 before spatial similarity comparison. The potential misregistration in $\mathrm{SN}_{\text {hyper }}$ regions may result in an underestimation of the spatial overlap between $\mathrm{SN}_{\text {hyper }}$ of the two modalities. Although the theoretical similarity between them has been discussed in detail, studies using the exact same spatial resolution could validate this conclusion more intuitively. Second, the $\mathrm{SN}_{\text {hyper }}$ regions were manually segmented. In PD patients with decreased neuromelanin, the difficulties in delineating this area could be a cause of relatively less DSC value in PD than $\mathrm{HC}$ group. Although the segmentation was conducted by two experienced radiologists with high ICC, an automated and objective segmentation method could be beneficial for future studies.

\section{Conclusion}

We proposed a setMag image derived from multiple short-echo-time magnitude images of QSM data, and quantitatively demonstrated that the setMag image could provide neuromelanin contrast by showing substantially similar location with SNc on NM-MRI, and the volume of this hyperintensity area could be used as a promising imaging biomarker for PD diagnosis. Our results show proofs for a new QSM-based multi-contrast imaging strategy, which could provide additional convenience in future PD studies.

\section{Abbreviations \\ SN: Substantia nigra; SNc: Substantia nigra pars compacta; $S N_{\text {hyper: }}$ Hyper intensity areas of substantia nigra; NM-MRI: Neuromelanin-sensitive MRl; QSM: Quantitative susceptibility mapping; setMag: Short echo time magnitude image; PD: Parkinson's disease; HCs: Healthy controls}

\section{Acknowledgments}

Authors thank Kristina Zeljic for her support in polishing the manuscript. Authors thank all patients and healthy controls in this study. All authors declare no conflicts of interest.

\section{Authors' contributions}

$L Q Y$ and $Y X L$ contributed to the study concept and design, and statistical analysis; $X L L$ contributed to the acquisition of data and a major contributor in writing the manuscript. PY W, Y Z, H Z and YH S contributed to the image post-processing. FT L and J W contributed to clinical assessments; DY G contributed to the study supervision and critical revision of the manuscript for important intellectual content. All authors contributed to revision of manuscript prior to submission for publication. All authors read and approved the final manuscript.

\section{Funding}

This study was funded by Science and Technology Commission of Shanghai Municipality [19ZR1407900, 17411953700], National Natural Science

Foundation of China [61672236], Ministry of Science and Technology of the
People's Republic of China [2016YFC1306500, 2016YFC1306504] and Huashan Hospital Fudan University Scientific Research project [2016QD085]. Funding agencies had no influence on the design of the study and collection, analysis, and interpretation of data and in writing the manuscript.

\section{Availability of data and materials}

The datasets used and analyzed during the current study are available from the corresponding author on reasonable request.

\section{Ethics approval and consent to participate}

This study was approved by the Ethics Committee of Huashan Hospital, Fudan University (approval No. KY 2016-214). Written consent was obtained from each participant.

\section{Consent for publication}

Not applicable.

\section{Competing interests}

None of the authors have any sources of support or conflicts of interests with regards to this article.

\section{Author details}

'Department of Radiology, Huashan Hospital, Fudan University, Shanghai 200040, China. ${ }^{2}$ Institute of Functional and Molecular Medical Imaging, Fudan University, Shanghai 200040, China. ${ }^{3}$ Department of Neurology, Huashan Hospital, Fudan University, Shanghai 200040, China. ${ }^{4}$ GE Healthcare China, Beijing 100176, China. ${ }^{5}$ Shanghai Key Laboratory of Medical Imaging Computing and Computer-Assisted Intervention, School of Basic Medical Sciences, Fudan University, Shanghai 200032, China.

Received: 12 February 2020 Accepted: 11 June 2020

Published online: 30 June 2020

\section{References}

1. Sasaki M, Shibata E, Tohyama K, Takahashi J, Otsuka K, Tsuchiya K, Takahashi $S$, Ehara S, Terayama Y, Sakai A. Neuromelanin magnetic resonance imaging of locus ceruleus and substantia nigra in Parkinson's disease. Neuroreport. 2006;17(11):1215-8.

2. Chen X, Huddleston DE, Langley J, Ahn S, Barnum CJ, Factor SA, Levey Al, Hu X. Simultaneous imaging of locus coeruleus and substantia nigra with a quantitative neuromelanin MRI approach. Magn Reson Imaging. 2014;32(10): $1301-6$.

3. Kitao S, Matsusue E, Fujii S, Miyoshi F, Kaminou T, Kato S, Ito H, Ogawa T. Correlation between pathology and neuromelanin MR imaging in Parkinson's disease and dementia with Lewy bodies. Neuroradiology. 2013; 55(8):947-53.

4. Zecca L, Stroppolo A, Gatti A, Tampellini D, Toscani M, Gallorini M, Giaveri G, Arosio P, Santambrogio P, Fariello RG, Karatekin E, Kleinman MH, Turro N, Hornykiewicz O, Zucca FA. The role of iron and copper molecules in the neuronal vulnerability of locus coeruleus and substantia nigra during aging. Proc Natl Acad Sci U S A. 2004;101(26):9843-8.

5. Guan X, Xu X, Zhang M. Region-specific Iron measured by MRI as a biomarker for Parkinson's disease. Neurosci Bull. 2017;33(5):561-7.

6. He N, Ling H, Ding B, Huang J, Zhang Y, Zhang Z, Liu C, Chen K, Yan F. Region-specific disturbed iron distribution in early idiopathic Parkinson's disease measured by quantitative susceptibility mapping. Hum Brain Mapp. 2015;36(11):4407-20.

7. Takahashi H, Watanabe $Y$, Tanaka H, Mihara M, Mochizuki H, Liu T, Wang Y, Tomiyama N. Quantifying changes in nigrosomes using quantitative susceptibility mapping and neuromelanin imaging for the diagnosis of early-stage Parkinson's disease. Br J Radiol. 2018;91(1086):20180037.

8. Takahashi H, Watanabe Y, Tanaka H, Mihara M, Mochizuki H, Takahashi K, Yamamoto K, Liu T, Wang Y, Tomiyama N. Comprehensive MRI quantification of the substantia nigra pars compacta in Parkinson's disease. Eur J Radiol. 2018;109:48-56.

9. Langley J, Huddleston DE, Chen X, Sedlacik J, Zachariah N, Hu X. A multicontrast approach for comprehensive imaging of substantia nigra. Neuroimage. 2015;112:7-13.

10. Langkammer C, Schweser F, Krebs N, Deistung A, Goessler W, Scheurer E, Sommer K, Reishofer G, Yen K, Fazekas F, Ropele S, Reichenbach JR. 
Quantitative susceptibility mapping (QSM) as a means to measure brain iron? A post mortem validation study. Neuroimage. 2012;62(3):1593-9.

11. Postuma RB, Berg D, Stern M, Poewe W, Olanow CW, Oertel W, Obeso J, Marek K, Litvan I, Lang AE, Halliday G, Goetz CG, Gasser T, Dubois B, Chan P, Bloem BR, Adler CH, Deuschl G. MDS clinical diagnostic criteria for Parkinson's disease. Movement Disord. 2015;30(12):1591-601.

12. Goetz CG, Tilley BC, Shaftman SR, Stebbins GT, Fahn S, Martinez-Martin P, Poewe W, Sampaio C, Stern MB, Dodel R, Dubois B, Holloway R, Jankovic J, Kulisevsky J, Lang AE, Lees A, Leurgans S, PA LW, Nyenhuis D, Olanow CW Rascol O, Schrag A, Teresi JA, van Hilten JJ, LaPelle N. Movement Disorder Society-sponsored revision of the Unified Parkinson's Disease Rating Scale (MDS-UPDRS): scale presentation and clinimetric testing results. Movement Disord. 2008;23(15):2129-70.

13. Goetz CG, Poewe W, Rascol O, Sampaio C, Stebbins GT, Counsell C, Giladi N, Holloway RG, Moore CG, Wenning GK, Yahr MD, Seidl L. Movement Disorder Society task force report on the Hoehn and Yahr staging scale: status and recommendations. Movement Disord. 2004;19(9):1020-8.

14. Liu C, Li W, Tong KA, Yeom KW, Kuzminski S. Susceptibility-weighted imaging and quantitative susceptibility mapping in the brain. J Magn Reson Imaging. 2015;42(1):23-41.

15. Yushkevich PA, Piven J, Hazlett HC, Smith RG, Ho S, Gee JC, Gerig G. Userguided 3D active contour segmentation of anatomical structures: significantly improved efficiency and reliability. Neuroimage. 2006;31(3):1116-28.

16. Zou K, Warfield S, Bharatha A, Tempany C, Kaus M, Haker S, Wells W, Jolesz F, Kikinis R. Statistical validation of image segmentation quality based on a spatial overlap index. Acad Radiol. 2004;11(2):178-89.

17. DeLong ER, DeLong DM, Clarke-Pearson DL. Comparing the areas under two or more correlated receiver operating characteristic curves: a nonparametric approach. Biometrics. 1988;44(3):837-45.

18. Landis JR, Biometrics GGKJ. The measurement of observer agreement for categorical data. Biometrics. 1977;33(1):159-74

19. Zecca L, Bellei C, Costi P, Albertini A, Monzani E, Casella L, Gallorini M, Bergamaschi L, Moscatelli A, Turro NJ, Eisner M, Crippa PR, Ito S, Wakamatsu K, Bush WD, Ward WC, Simon JD, Zucca FA. New melanic pigments in the human brain that accumulate in aging and block environmental toxic metals. Proc Natl Acad Sci U S A. 2008;105(45):17567-72.

20. Trujillo P, Summers PE, Ferrari E, Zucca FA, Sturini M, Mainardi LT, Cerutti S, Smith AK, Smith SA, Zecca L, Costa A. Contrast mechanisms associated with neuromelanin-MRI. Magn Reson Med. 2017;78(5):1790-800.

21. Tosk JM, Holshouser BA, Aloia RC, Hinshaw DB, Hasso AN, MacMurray JP Will AD, Bozzetti LP. Effects of the interaction between ferric iron and $L$ dopa melanin on $\mathrm{T} 1$ and $\mathrm{T} 2$ relaxation times deter mined by magnetic resonance imaging. Magn Reson Med. 1992;26(1):40-5.

22. Henkelman RM, Stanisz GJ, Graham SJ. Magnetization transfer in MRl: a review. NMR Biomed. 2001;14(2):57-64.

23. Zucca FA, Segura-Aguilar J, Ferrari E, Munoz P, Paris I, Sulzer D, Sarna T, Casella $L$, Zecca L. Interactions of iron, dopamine and neuromelanin pathways in brain aging and Parkinson's disease. Prog Neurobiol. 2017;155:96-119.

24. Zecca L, Shima T, Stroppolo A, Goj C, Battiston G, Gerbasi R, Sarna T, Swartz $H$. Interaction of neuromelanin and iron in substantia nigra and other areas of human brain. Neuroscience. 1996;73(2):407-15.

25. Taniguchi D, Hatano T, Kamagata K, Okuzumi A, Oji Y, Mori A, Hori M, Aoki S, Hattori N. Neuromelanin imaging and midbrain volumetry in progressive supranuclear palsy and Parkinson's disease. Movement Disord. 2018;33(9):1488-92.

26. Castellanos G, Fernandez-Seara MA, Lorenzo-Betancor O, Ortega-Cubero S, Puigvert M, Uranga J, Vidorreta M, Irigoyen J, Lorenzo E, Munoz-Barrutia A, Ortiz-de-Solorzano C, Pastor P, Pastor MA. Automated neuromelanin imaging as a diagnostic biomarker for Parkinson's disease. Movement Disord. 2015;30(7):945-52.

27. Schwarz ST, Xing Y, Tomar P, Bajaj N, Auer DP. In vivo assessment of brainstem depigmentation in Parkinson disease: potential as a severity marker for multicenter studies. Radiology. 2017;283(3):789-98.

28. Fabbri M, Reimão $S$, Carvalho M, Nunes R, Abreu D, Guedes L, Bouça R, Lobo P, Godinho C, Coelho M, Gonçalves N, Rosa M, Antonini A, Ferreira J. Substantia Nigra Neuromelanin as an imaging biomarker of disease progression in Parkinson's disease. J Park Dis. 2017;7(3):491-501.

\section{Publisher's Note}

Springer Nature remains neutral with regard to jurisdictional claims in published maps and institutional affiliations.

\section{Ready to submit your research? Choose BMC and benefit from:}

- fast, convenient online submission

- thorough peer review by experienced researchers in your field

- rapid publication on acceptance

- support for research data, including large and complex data types

- gold Open Access which fosters wider collaboration and increased citations

- maximum visibility for your research: over $100 \mathrm{M}$ website views per year

At BMC, research is always in progress.

Learn more biomedcentral.com/submissions 